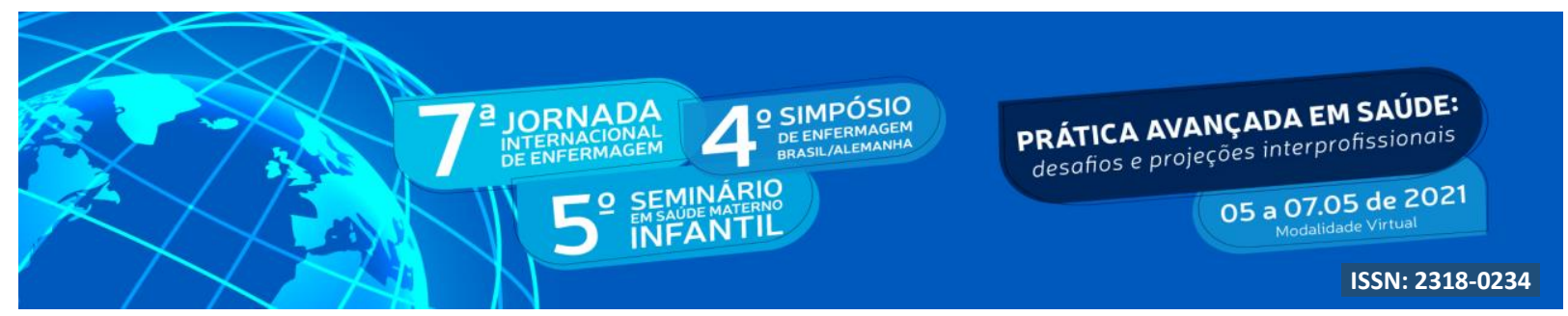

DOI: http://doi.org/10.48195/jie2021-148

\title{
REDE SOCIAL COMO FERRAMENTA PEDAGÓGICA PARA EDUCAR EM SAÚDE: RELATO DE EXPERIÊNCIA
}

\author{
Angelo Ramos Junior'1; Oclaris Lopes Munhoz ${ }^{2}$; Diego Schaurich ${ }^{3}$; Zaira Letícia Tisott ${ }^{4}$ \\ ; Nagianne Oliveira dos Santos ${ }^{5}$; Cláudia Zamberlan ${ }^{6}$
}

\begin{abstract}
RESUMO
A rede social Facebook é uma ferramenta amplamente utilizada na disseminação de informações e, no contexto da saúde, pode ser utilizada com potencial de inovação na construção do conhecimento popular e científico. Destaca-se que o Brasil é o país com o segundo maior número de usuários desta rede social no mundo, atrás apenas dos Estados Unidos da América. Assim, objetiva-se relatar a participação de um profissional enfermeiro em uma Live via Facebook sobre a temática de infecção pelo novo coronavírus. Trata-se de um relato de experiência a partir do desenvolvimento de Live via Facebook, realizada em março de 2019 , e que possui aproximadamente 10 mil visualizações e 200 compartilhamentos. Esta experiência demonstra que as redes sociais apresentam um significativo potencial para ser utilizado como ferramenta pedagógica no ensino acadêmico e popular.
\end{abstract}

Palavras-chave: Educação em Saúde; Rede Social; Saúde Pública.

\begin{abstract}
The social network Facebook is a tool widely used in the dissemination of information and, in thecontext of health, it can be used with potential for innovation in the construction of popular and scientific knowledge. It is noteworthy that Brazil is the country with the second largest number of users of this social network in the world, behind only the United States of America. Thus, the objective is to report the participation of a professional nurse in a Live via Facebook on the theme of infection with the new coronavirus. This is an experience report from the development of Live via Facebook, held in March 2019, which has approximately 10,000 views and 200 shares. This

\footnotetext{
${ }^{1}$ Enfermeiro. Mestre em Enfermagem. Sistema de Ensino Gaúcho. E-mail: angeloramosjunior@gmail.com ${ }^{2}$ Enfermeiro. Doutorando em Enfermagem. Universidade Federal de Santa Maria. E-mail: oclaris_munhoz@hotmail.com

${ }^{3}$ Enfermeiro. Doutorando em Educação. Universidade Federal de Santa Maria. E-mail: eu_diegosch@hotmail.com

${ }^{4}$ Enfermeira. Doutoranda em Enfermagem. Universidade Federal do Rio Grande do Sul. E-mail: zairatisott10@gmail.com

${ }^{5}$ Enfermeira. Residente em Infectologia e Neurologia. Universidade Franciscana. E-mail: ng.oliver@hotmail.com

${ }^{6}$ Enfermeira. Doutora em Enfermagem. Universidade Franciscana. E-mail: claudiaz@ufn.edu.br
} 


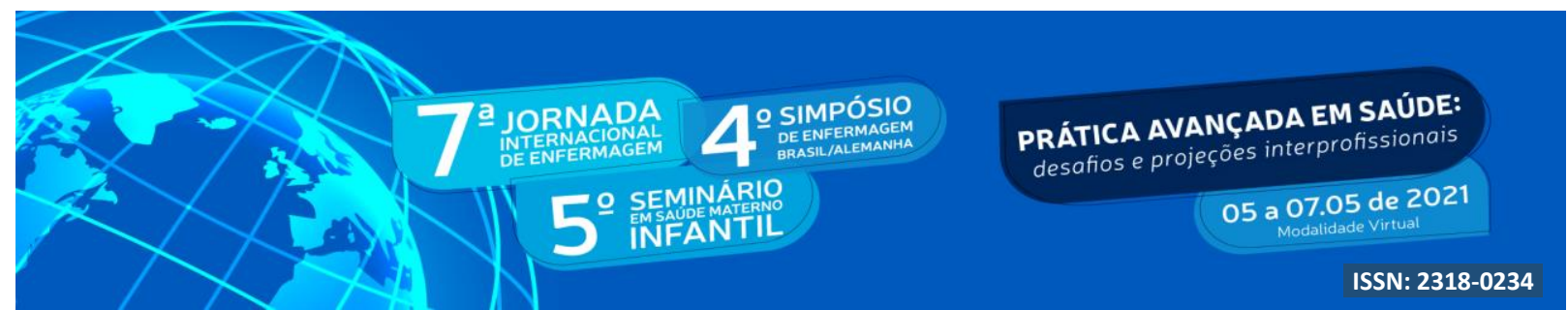

experience demonstrates that social networks have significant potential to be used as a pedagogical tool in academic and popular education.

Key Words: Health education; Social network; Public health.

\section{INTRODUÇÃO}

A pandemia do novo coronavírus, causador da COVID-19, explicita a necessidade de reafirmar a participação das redes sociais no processo de educar e compartilhar saberes em tempos de distanciamento físico. Diante do contexto atual, o uso da tecnologia como ferramenta de disseminação de conhecimentos direciona para a possibilidade de unir população e profissionais da saúde (BERNARDES et al., 2019).

Inúmeras são as redes sociais existentes, algumas com caráter mais educativo, outras com características lúdicas e há àquelas que agregam os dois elementos. Uma das redes sociais efetivamente acessada pela população mundial é o Facebook, a qual se configura como importante instrumento na construção de conhecimentos populares e científicos em saúde, além de ser muito utilizada no Brasil, país com o segundo maior número de usuários no mundo, atrás apenas dos Estados Unidos da América. Desse modo, o seu uso pode ser positivo nas ações de educação em saúde, o que fortalece a importância de utilizar as redes sociais como possibilidade potencial de aprendizados, especialmente em momentos como o atual: de pandemia (ARAGÃO et al., 2017).

Neste sentido, o profissional da saúde é um importante ator fornecedor de informações baseadas em evidências científicas, destacando-se que a inserção de novas possibilidades educativas e pedagógicas são desafiadoras para que o conhecimento e a informação de qualidade sejam compartilhados. Somado a isso, o uso de linguagem acessível e de fácil entendimento possibilita a compreensão e a relação entre pessoas, sendo que o uso do Facebook favorece o acesso a estas informações (NASS et al., 2019). 


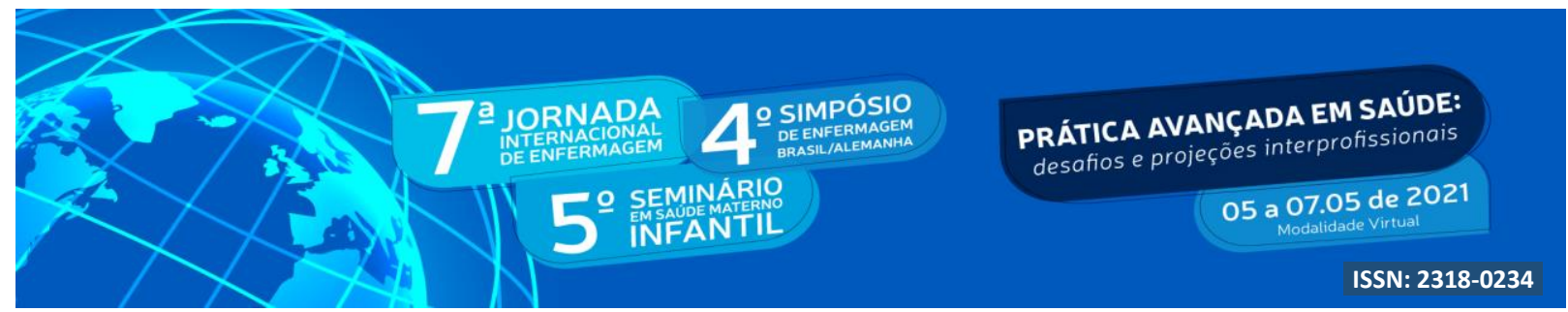

Diante disso, a realização de Lives via redes sociais como ferramenta de educar em saúde corrobora para a disseminação de informações/orientações adequadas e, também, para a qualificação científica profissional, o que vem ao encontro da ciência, dos profissionais e da população em geral.

\section{OBJETIVO}

Relatar a participação de um profissional enfermeiro em uma Live via Facebook sobre a temática de infecção pelo novo coronavírus.

\section{METODOLOGIA}

Trata-se de um relato de experiência referente a uma Live desenvolvida em rede social, via Facebook, com vistas a fornecer esclarecimentos e orientações sobre a pandemia do novo coronavírus, causador da doença COVID-19. Essa Live foi realizada na cidade de Santa Maria, Rio Grande do Sul, Brasil, no mês de março de 2019 e contou com aproximadamente 10 mil visualizações e 200 compartilhamentos.

A educação em saúde por meio da Live ocorreu a partir da proposta de uma advogada do município de Santa Maria, Rio Grande do Sul, Brasil, que trabalha com populações vulneráveis. Assim, aconteceu uma divulgação prévia desta ação, com 24 horas de antecedência da Live, em redes sociais.

A Live teve duração de 60 minutos e está disponível em rede social Facebook (link nas referências) e no Youtube (link nas referências). Torna-se importante destacar que, após a finalização da transmissão ao vivo, as perguntas foram respondidas via chat, e foram realizadas orientações alinhadas aos protocolos referendados, até aquele momento, por órgãos científicos nacionais e internacionais relacionados à COVID-19. 


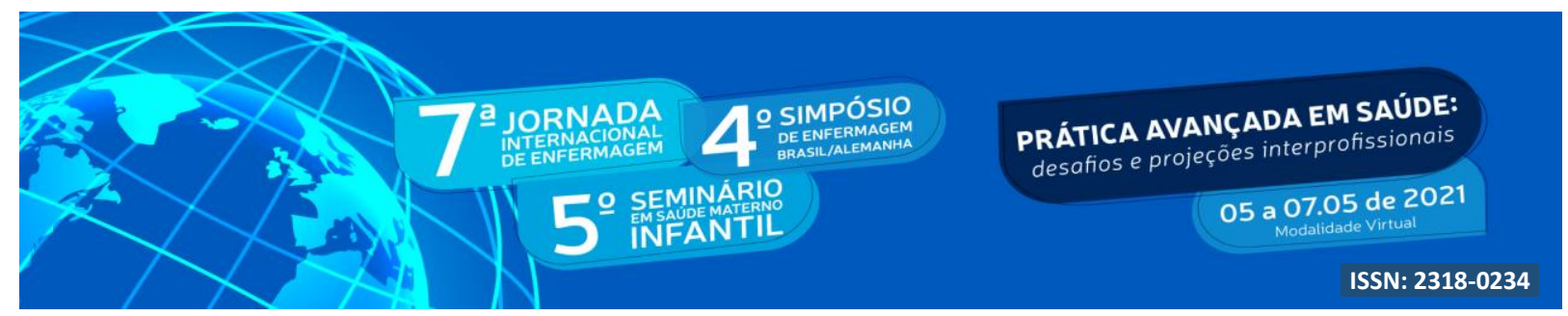

\section{RESULTADOS E DISCUSSÃO}

Durante a transmissão, a população residente no Estado do Rio Grande do Norte enviou perguntas para serem respondidas, fato que demonstra a abrangência, além, do estado do Rio Grande do Sul da tecnologia utilizada para transmissão da Live. Ressalta-se que o número de visualizações extrapolou as expectativas, o que pode ser justificado por ser uma temática emergente até então e, portanto, com relativa escassez de informações científicas.

A entrevista foi concedida por um enfermeiro infectologista com conhecimento na área de doenças infectocontagiosas, que respondeu as perguntas enviadas pelos espectadores, bem como as perguntas elaboradas previamente pela advogada entrevistadora.

Frente ao exposto, considera-se que o trabalho desempenhado pelo profissional da saúde é dinâmico e multifatorial, e necessita de novas possibilidades de acesso a infomações relacionadas à pandemia do novo coronavírus, uma vez que essas informações precisam ser acessadas por todas as classes sociais. Além disso, o profissional precisa se adaptar às diferentes formas de disseminação desse conhecimento, sendo que uma das maneiras para que isso ocorra de modo científico e direcionado às melhores evidências é com a utilização das tecnologias e das redes sociais disponíveis.

Para tanto, educar para saúde utilizando as redes sociais é uma das ações mais acessíveis pela facilidade que essas ferramentas apresentam, quais sejam: diversidade de fotos e vídeos, possibilidade de desenvolvimento de intervenções que impactam positivamente na vida das pessoas e pelo fato de serem exploradas como um importante instrumento pedagógico para a disseminação do conhecimento (SCHERER e FARIAS, 2018). Ademais, o uso do Facebook passou a ser disponibilizado de maneira gratuita, o que inclui pessoas com vulnerabilidade socioeconômica, tornando-a uma ferramenta inclusiva.

Estudo de Martins e Gouveia (2019), realizado com 26 estudantes utilizando um grupo criado no Facebook, obteve como resultado que a interação e a participação nessa ferramenta foi mais efetiva que as utilizadas anteriormente pelos docentes. Também, a maioria dos participantes do estudo sentiram-se satisfeitos com a possibilidade de construir conhecimentos a partir de uma ferramenta familiar aos mesmos. 


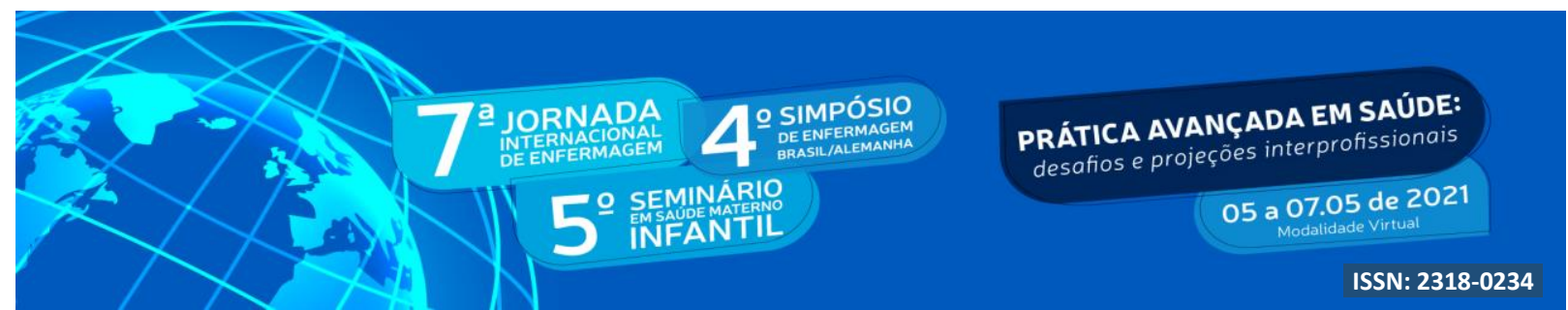

Nessa perspectiva, e contextualizando a importância da utilização de novas possibilidades de ensino e aprendizagem científico e popular, destaca-se o estudo realizado no Estado do Ceará com graduandos de enfermagem, o qual possibilitou a manutenção de vínculo entre os colegas durante o distanciamento físico, bem como o compartilhamento de notícias atuais para a obtenção de novos conhecimentos. Nesse sentido, os estudantes relataram que a utilização dessa rede social possibilitou a comunicação efetiva durante o período de isolamento decorrente da pandemia do novo coronavírus (CHAVES; BARBOSA; NÓBREGA-THERRIEN, 2020).

\section{CONCLUSÃO}

O estudo mostrou que as redes sociais, em especial, o Facebook, apresentam um significativo potencial para serem utilizadas como ferramenta pedagógica no ensino acadêmico e, também, para a disseminação de informações/orientações embasadas em conhecimentos técnico-científicos para a população em geral. Esse fato estimula e mantém a motivação das pessoas em um período de distanciamento físico, o que torna a utilização dessa ferramenta essencial para o desenvolvimento e o aprimoramento dos conhecimentos, sejam conhecimentos populares ou conhecimentos acadêmicos.

Assim, há a necessidade de publicação de novos estudos que abrangem a eficiência da utilização da rede social para a disseminação de conhecimentos no sentido de compreender que as ferramentas que estão disponíveis podem ser potentes quando utilizadas de forma coerente e científica. 


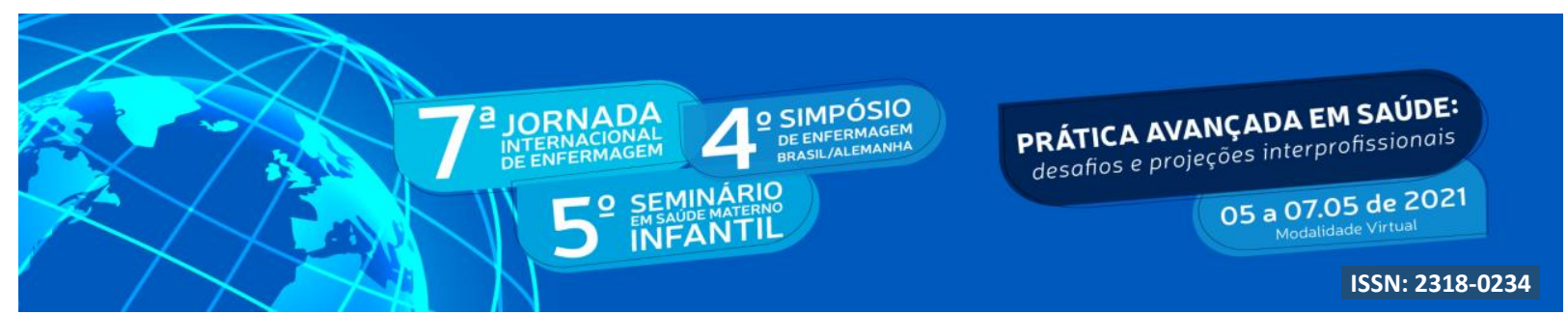

\section{REFERÊNCIAS}

ARAGÃO, J. M. N. et al. O uso do Facebook na aprendizagem em saúde: percepções de adolescentes escolares. Rev Bras Enferm. v. 71. n. 2. p.286-92. 2018.

BERNARDES, V. P. et al. Facebook® como ferramenta pedagógica em saúde coletiva: Integrando Formação Médica e Educação em Saúde. Revista Brasileira de Educação Médica, v. 43, n. 1, p. 652-661, 2019.

MARTINS, E.; GOUVEIA, L. Facebook como Ferramenta de Apoio ao Ensino. In: Anais da X Escola Regional de Informática de Mato Grosso. SBC, 2019. p. 148-150.

NASS, E. M. A. et al. Perspectiva de jovens com diabetes sobre intervenção educativa na rede social Facebook®. Acta Paulista de Enfermagem, v. 32, n. 4, p. 390-397, 2019.

Live. Disponível em: Facebook: https://www.facebook.com/marinamcallegaro/videos/254377602243439/ $\quad$ Youtube: https://youtu.be/-WuAFUFipdo

SCHERER, A. L.; DE FARIAS, J. G. Uso da Rede Social Facebook como Ferramenta de Ensino-aprendizagem em Cursos de Ensino Superior. Revista Brasileira de Aprendizagem Aberta e a Distância, v. 17, n. 1, 2018. 